\title{
Air temperature stratification when flowing between rooms
}

\author{
Samvel Sargsyan*, and Anna Tsap \\ Moscow State University of Civil Engineering, Yaroslavskoe shosse, 26, Moscow, 129337, Russia
}

\begin{abstract}
The article presents the regularities of the vertical change in the air temperature of a ventilated room for various purposes, where the excess of sensible heat dominates, in the first case, when there is no process of air flow through the opening in a common vertical fence between the rooms located in a row, or the second case, when a similar air flow is observed. In heat-stressed rooms, the general ventilation arrangement scheme provides for the removal of air from the upper volume of the room. To determine the required air exchange with point, two-zone or multi-zone mathematical models of a ventilated room, it is necessary to have a reliable apparatus for determining the gradient of the vertical air parameters change for a ventilated room. The reasonably determined value of the gradient of the air temperature change along the vertical of the ventilated room makes it possible to calculate the air flow between different volumes inside the ventilated room. Determination of the air temperature near the suction flare makes it possible to determine the required air exchange more reasonably. A method is given for calculating the distribution of air temperature in rooms with an excess of apparent heat over the height of the room in the first case, when there is no process of air flow through the opening in the common vertical fence between the rooms located in a row, or the second case, when a similar air flow is observed. This takes into account the height of the supply air distributors relative to the working (serviced) area. Reasonable determination of the air temperature at different points in the room with an excess of sensible heat allows a correct calculation of the required air exchange. The presented method for determining the gradient of air temperature stratification along the height of the room with air flow between adjacent rooms and without it contributes to the reasonable determination of the air temperature at the suction flame of the exhaust air distributor, which increases the reliability of calculating the required air exchange.
\end{abstract}

\section{Introduction}

A heat-intensive room is considered, where the excess of sensible heat with a stationary airthermal regime dominates, with known capacities, with given locations of heat sources, where the supply and exhaust mechanical ventilation system operates, providing a balance in the amount of supply $\left(G_{p}, k g / h\right)$ and extract air $\left(G_{u}, k g /\right.$ hour $) G_{p}=G_{u}$. The stratification of air temperature inside a ventilated room is caused by the flow of sensible

${ }^{*}$ Corresponding author: sargsyansv@mgsu.ru 
heat from solar radiation, from service personnel, from technological equipment and from other sources of heat release inside the room under consideration. The process of air temperature stratification along the vertical of a ventilated room is influenced by the processes of mixing air inside the room by supply ventilation jets, the processes of infiltration or exfiltration, as well as the flow of air between the rooms located in a row. Cases are considered when the supply ventilation stream enters the working (serviced) area from the upper zone of the ventilated room or the ventilation stream enters the working (serviced) area. In all cases, the ventilation stream dissipates within the working (serviced) area.

To determine the required air exchange with point, two-zone or multi-zone mathematical models of a ventilated room, it is necessary to have a reliable apparatus for determining the gradient of the vertical air parameters change for a ventilated room. The reasonably determined value of the gradient of the air temperature change along the vertical of the ventilated room makes it possible to calculate the air flow between different volumes inside the ventilated room.

Determination of the air temperature near the suction flare makes it possible to determine the required air exchange more reasonably [1-20].

\section{Derivation of a formula characterizing stratification}

To compile a mathematical model of heat and mass transfer processes in a ventilated room with an excess of apparent heat, it is required to determine the patterns of change in the air temperature gradient along the vertical of the ventilated room in the absence of an overflow process (Figure 1) and in the presence of a process of overflow into a parallel room through an opening in a vertical fence between them (Figure 2).

The volume of air in the supply stream when it enters the working (serviced) area at the level h (Figure 1), provided that the height of the working area (h) is less than the height of the air distribution grille $\left(\mathrm{H}_{\mathrm{p}}\right), \mathrm{h} \leq \mathrm{H}_{\mathrm{p}}$ is determined by the formula:

$$
G_{h}=G_{p} \cdot\{[\beta-(\beta-1) / \beta] \cdot h\},
$$

where: $\beta$ - is the dimensionless coefficient of increase in the air flow rate in the supply stream, associated with the suction of air by the supply stream and depending on the conditions of air outflow from the air distributor; $G_{p}$ is the amount of supply air; $h$ - is the height of the working (serviced) area.

Provided that $h>H_{p}$, where $H_{p}$ is the height of the supply air distributor less than the height of the working (serviced) zone, the air flow rate in the jet within the working (serviced) zone remains unchanged:

$$
G_{h}=G_{p}=\text { const }
$$

In Figure 2, a diagram of the organization of general ventilation is presented where air overflow through the opening in the vertical wall into the parallel room is observed in an organized manner. In the case when the process of air overflow into a parallel room occurs uniformly over the entire height of the vertical opening, and the supply air diffuser is located above the working (serviced) zone, that is, $h \leq \mathrm{H}_{\mathrm{p}}$, the amount of air in the vertical flow overflowing through the vertical opening is determined as:

$$
G_{h}=G_{p} \cdot\left\{\beta-\left[(\beta-1) / H_{p}+1 / H\right] \cdot h\right\},
$$


where: $G_{h}$ the amount of air in the vertical air flow moving through the opening towards the parallel space; $\mathrm{H}$ is the height of the room in question from floor level to the bottom of the ceiling.

When the supply air distributor is located in the volume of the working (serviced) zone, that is, $h>\mathrm{H}_{\mathrm{p}}$, the flow rate of the vertical airflow through the fence is determined as:

$$
G_{h}=G_{p} \cdot(1-h / H)
$$

The distribution of air temperature in a room with heat sources evenly spaced over the area of a heat-stressed room where the specific value of the thermal stress is $\mathrm{q}_{\mathrm{t}}\left(\mathrm{W} / \mathrm{m}^{3}\right)$ depends on the amount of air in the vertical flow flowing into a parallel room under the influence of ascending and descending convective jets.

Sratification of air temperature along the vertical of a ventilated room in the most general case is defined as:

$$
d t=d Q /\left(c_{6} \cdot G_{h}\right),
$$

Where: dQ - the amount of heat released in an elementary volume of a ventilated room, the height of which is dh with a base area $f_{p}$ :

$$
d Q=q_{t} \cdot f_{p} \cdot d h
$$

where: $\mathrm{q}_{\mathrm{t}}$ - heat release in the elementary volume of the room, with height $\mathrm{dh}$ and base area $f_{p}$ :
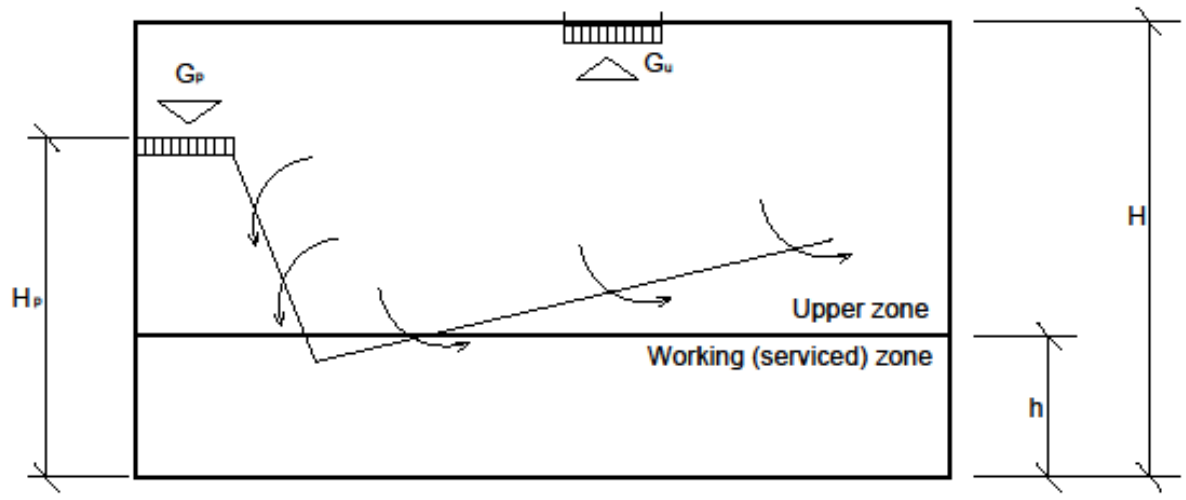

Fig. 1. Diagram of air flow distribution in a ventilated room in the absence of air flow through a vertical opening into a parallel room

The differential equation relating the value of the gradient of the change in air temperature along the vertical of the ventilated room in the presence of the process of air flow between parallel rooms and without overflow, when the supply air diffuser is located above the working (serviced) zone is determined by inserting the data from equations (1-3) and (6) into equation (5).

With a scheme without air flow into an adjacent room (Figure 1) and when the supply air diffuser is located above the working (serviced) zone $\left(\mathrm{h} \leq \mathrm{H}_{\mathrm{p}}\right)$, taking into account the above, an expression can be derived that allows to determine the gradient of the air temperature change along the height:

$$
d t=q_{t} \cdot f_{p} \cdot d h /\left(c_{b} \cdot G_{p}\right) \cdot\{[\beta-(\beta-1) / \beta] \cdot h\},
$$


Integrating expression (7) within the temperature range from $t_{v}\left(t_{v}\right.$ is the air temperature in the working or serviced area) to $t_{h}\left(t_{h}\right.$ is the air temperature at a height $h$ from the floor level), and in height from $\mathrm{h}=0$ to $\mathrm{H}$ (room height from floor level to the bottom of the slab), we obtain an analytical formula that allows us to determine the change in air temperature along the height of a ventilated room with excess sensible heat without air flow between adjacent rooms when the supply air diffuser is located above the working (serviced) zone $\left(\mathrm{h} \leq \mathrm{H}_{\mathrm{p}}\right)$ :

$$
t_{h}=t_{v}+\left[q_{t} \cdot f_{p} /\left(c_{\theta} \cdot G_{p}\right)\right] \cdot[H /(\beta-1)] \cdot \ln \left[\beta-h \cdot(\beta-1) / H_{p}\right],
$$

With an air distribution scheme with no air flow between adjacent rooms (Figure 1) and when the supply air diffuser is located in the volume of the working (serviced) zone ( $\mathrm{h}>$ $\mathrm{H}_{\mathrm{p}}$ ), we obtain an expression that allows us to determine the gradient of the air temperature change in height:

$$
d t=q_{t} \cdot f_{p} \cdot d h /\left(c_{6} \cdot G_{p}\right)
$$

Integrating expression (9) within the temperature range from $t_{v}\left(t_{v}\right.$ is the air temperature in the working or serviced area) to $\mathrm{t}_{\mathrm{Hp}}\left(\mathrm{t}_{\mathrm{Hp}}\right.$ is the air temperature at the installation height of the supply air distributor), and in height from $\mathrm{h}=0$ to $\mathrm{H}$ (the room height from the level floor to the bottom of the floor), we obtain an analytical formula that allows us to determine the change in air temperature along the height of a ventilated room with an excess of sensible heat without air flow between adjacent rooms when the supply air distributor is located in the volume of the working (serviced) zone $\left(h>H_{p}\right)$ :

$$
t_{h}=t_{H_{p}}+\left[q_{t} \cdot f_{p} /\left(c_{b} \cdot G_{p}\right)\right] \cdot\left[h-H_{p}\right],
$$

where: $\mathrm{t}_{\mathrm{Hp}}$ - air temperature at the height of the air distributor, $\mathrm{H}_{\mathrm{p}}$ defined as:

$$
t_{H_{p}}=t_{v}+\left[q_{t} \cdot f_{p} \cdot H_{p} /\left(c_{b} \cdot G_{p}\right)\right] \cdot \ln \beta^{1 /(\beta-1)},
$$

Therefore, when the supply air distributor is located in the volume of the working area $\left(h \geq H_{p}\right.$ ), the change in air temperature along the height of the ventilated room with surplus of sensible heat without airflow between adjacent rooms is defined as:

$$
t_{H_{p}}=t_{v}+\left[q_{t} \cdot f_{p} \cdot H_{p} /\left(c_{\theta} \cdot G_{p}\right)\right] \cdot\left[h-H_{p} \cdot\left(1-\ln \beta^{1 /(\beta-1)}\right)\right],
$$

The air temperature removed from the upper point of the room $\left(t_{u}\right)$, in the absence of overflow between adjacent rooms (Figure 1), is determined as:

$$
t_{u}=t_{v}+\left[q_{t} \cdot f_{p} /\left(c_{6} \cdot G_{p}\right)\right] \cdot\left[H-H_{p} \cdot\left(1-\ln \beta^{1 /(\beta-1))}\right]\right.
$$

With an air distribution scheme in rooms with an excess of sensible heat, where there is air flow between adjacent rooms (Figure 2) and when the supply air diffuser is located above the volume of the working (serviced) zone $\left(h \leq \mathrm{H}_{\mathrm{p}}\right)$, we obtain an expression that allows us to determine the gradient of the air temperature change by height:

$$
t_{h}=t_{v}+\left[q_{t} \cdot f_{p} /\left(c_{\theta} \cdot G_{p}\right)\right] \cdot\left[H_{p} /\left(H_{p} / H+\beta-1\right)\right] \cdot \ln \left[\beta /\left(\beta-h / H_{p} \cdot\left(H_{p} / H+\beta-1\right],(14\right.\right.
$$


In similar conditions with air overflow, when the height of the supply air distributor is at the level of the working (serviced) zone, that is: $h=\mathrm{H}_{\mathrm{p}}$, the air temperature at the air distributor level is determined by the analytical formula:

$$
t_{H_{p}}=t_{v}+\left[q_{t} \cdot f_{p} /\left(c_{6} \cdot G_{p}\right)\right] \cdot\left[H /\left(H_{p} / H+\beta-1\right)\right] \cdot \ln \left[\left(1-H_{p} / H\right) /(1-h / H)\right],
$$

With the air distribution scheme in rooms with an excess of sensible heat, where there is air flow between adjacent rooms (Figure 2), and when the supply air diffuser is located in the volume of the working (serviced) zone $\left(h \geq H_{p}\right)$, taking into account expression (14), we obtain the formula, allowing to determine the gradient of air temperature change along the height:

$$
t_{h}=t_{H_{p}}+\left[q_{t} \cdot f_{p} /\left(c_{6} \cdot G_{p}\right)\right] \cdot H \cdot \ln \left[\left(1-H_{p} / H\right) /(1-h / H)\right]
$$

The resulting expression (16) is special in that when the height of the working (serviced) area for technological reasons increases and tends to reach the height of the room as a whole $(\mathrm{h} \rightarrow \mathrm{H})$, the value of the second term tends to infinity. Therefore, the temperature at the highest altitude will be the highest.

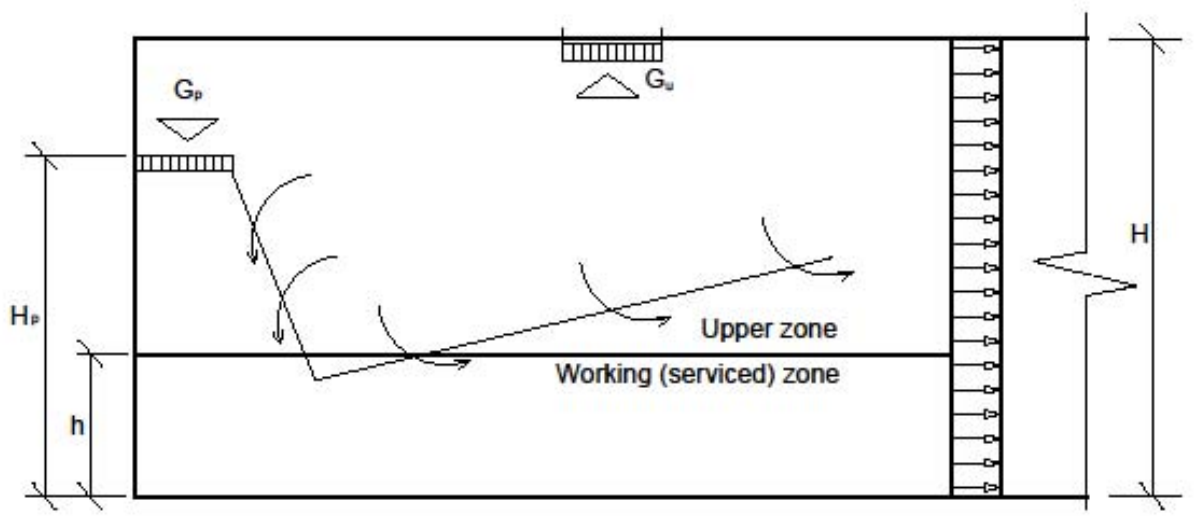

Fig. 2. Diagram of the distribution of air flows in a ventilated room in the presence of air flow into an adjacent room.

Actual temperatures in the under-ceiling space of a ventilated room can be very high, tending to the temperature of the surfaces of heat sources. The presence of air overflow through the opening in the vertical fence between the parallel rooms leads to overheating of the air in the under-ceiling area of the ventilated room, the overflow in the upper part of the vertical opening increases, which contributes to the flow of air into the ventilated volume from the adjacent room, through the lower part of the vertical opening. 


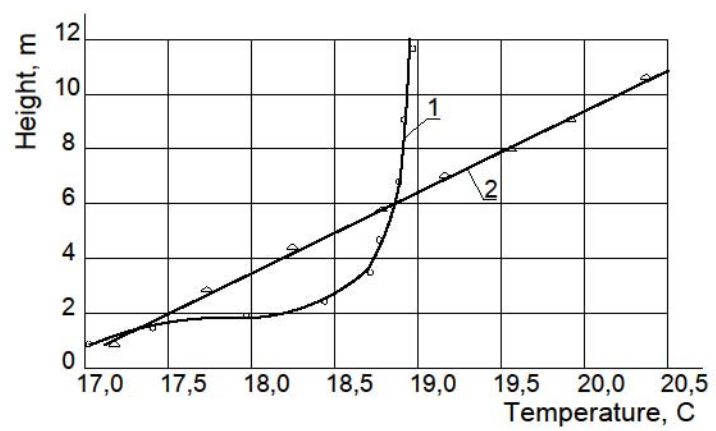

Fig. 3. Air temperature stratification along the vertical of a ventilated room with obvious thermal excesses, when the supply air is supplied to the upper zone $\left(\mathrm{h} \leq \mathrm{H}_{\mathrm{p}}\right)$ in the presence of air overflow into a parallel room (dependence 2 ) and without overflow (dependence 1)

\section{Results}

When the amount of air removed from the ventilated room is equal to $0.1 \times \mathrm{x}_{\mathrm{p}}$, the amount of air flowing through the vertical opening into the parallel room will be equal to $0.9 \mathrm{xG}_{\mathrm{p}}$. Then, using equation (16), with a sufficient degree of accuracy, we obtain the result corresponding to real conditions.

Figure 3 graphically presents the results of calculations of a particular case. Where are the changes in air temperature along the height of a heat-stressed room with air flow between adjacent rooms and without it. Initial data for the calculation: room dimensions $12 \times 12 \times 12(h) \mathrm{m}$; height of the working area $-\mathrm{h}=2 \mathrm{~m}$; height of the supply air distributor $\mathrm{H}_{\mathrm{p}}$ $=5 \mathrm{~m}$; heat and intensity of the room $\mathrm{q}_{\mathrm{t}}=90\left(\mathrm{~W} / \mathrm{m}^{3}\right)$; supply air consumption $-\mathrm{L}_{\mathrm{p}}=$ $4000 \mathrm{~m}^{3} / \mathrm{h}$. Figure 3 shows the results of calculations in graphical form.

Figure 3 shows graphical dependencies of air temperature stratification along the vertical of a ventilated room where technological sources of heat release are evenly distributed over the area of the ventilated room in the first case, when there is no process of air flow through the opening in the common vertical fence between the rooms located in a row and the second case, when a similar is observed overflow of air.

\section{Conclusions}

The obtained expressions make it possible to determine the gradient of the change in air temperature in rooms with an excess of apparent heat along the vertical of the ventilated room, depending on the location of the supply air distributor in the presence of air flow between parallel rooms and without overflow.

The ability to determine the flow rate and temperature of the air flowing into a parallel room is necessary when drawing up balance equations for a two-zone mathematical model of heat and mass transfer processes in a ventilated room.

A substantiated calculation of the required air exchange using a two-zone mathematical model of a ventilated room is possible with a reasonable determination of the air temperature, taking into account stratification, at different points in the volume of the room where the excess of sensible heat dominates.

The proposed method for calculating the gradient of air temperature stratification along the vertical of a ventilated room with air flow between adjacent rooms and without it contributes to the reasonable determination of the air temperature at the suction flame of the 
exhaust air distributor, which increases the reliability of calculating the required air exchange.

\section{References}

1. SP 60.13330.2012 Heating, ventilation and air conditioning (GUP TsPP, Moscow, 2012)

2. GOST 30494-2011 Residential and public buildings. The parameters of the micro climate in the premises. (Standardinform, Moscow, 2013)

3. V.N. Bogoslovskiy, Building Thermophysics (thermophysical basis of heating, ventilation and air conditioning) (Moscow, 2013)

4. S.V. Sargsyan, Methods of laboratory testing on physical models Scientific review, №16, 76-79 ( 2015 )

5. P. N. Kamenev, E. I. Tertichnik, Ventilation (ACB, Moscow, 2014)

6. S.V.Sargsyan, V.A.Borisova, Mathematical model of spatial heat exchange of rooms with linearly sources of heat, 4 Interhational multi-conference on industrial engineering and modern technologies, 2-4 October 2018, Vladivostok, Russia (2018)

7. V.P.Titov, S.V.Sargsyan, Bibliographical Index of deposed manuscripts, 5, 10910 (1991)

8. S.V. Sargsyan, Investigation of the ways to organize the air exchange and air difuusion systems on physical models in the laboratory conditions //.Scientific review, №16, 68$71(2015)$

9. V.A.Zhila, S.V. Sargsyan, Engineering systems of buildings and structures. Heat and ventilation (Moscow pub.Academia, 2014)

10. S V. Sargsyan, Jila VA On Modeling of Influence of Air Condition of Building on Gasconsuming Equipment with an Open Combustion Chamber Functioning

11. V.P Titov., Sargsyan S.V. This is a model for the ventilated space. - Dep. Vo VNIINTPI No. 10910, February 18, 1991., 1991, Bibliographical Index of deposed manuscripts, issue.5.

12. S.V. Sargsyan, A.G. Rymarov Materials and gas supply and ventilation systems, MGSU, Moscow, 23-25 November 2005, p. 147-149.

13. P.N. Kamenev, E.I. Tertichnik. Ventilation. - M.: Association of Construction Universities, 2006.

14. S.V. Sargsyan Methods of determination of the quantity and the characteristics of air diffusers located in the upper zone of the premise when the air is supplied by vertical jets. Scientific and technical revue MGSU Bulletin, Special edition 2/2009, MGSU, Moscow, p. 452-456.

15. V.P. Titov, S.V. Sargsyan Universal two-zone model of a space for calculation of the required air exchange/ in Collected works: Labor protection in industry. Penza, 1991.

16. S.V. Sargsyan, A.D. Spirin Calculation of the air exchange at the laboratory of fireresistance testing of construction elements and structures by the method of zone balances. Scientific and technical revue MGSU Bulletin, Issue 8/2014, MGSU, Moscow, p.

17. S. V. Sargsyan, V. A. Shalunova, Santekhnika, otopleniye, konditsionirovaniye, 2(218), 52-58 (2020)

18. E. G. Malyavina, S. V. Biryukov, AVOK, 2, 40-44 (2008)

19. S. V. Sargsyan, E.K. Muracheva, Nauchnoye obozreniye, 11, 35-38 (2017) 
20. S.V. Sargsyan Investigation of the ways to organize the air exchange and air difuusion systems on physical models in the laboratory conditions // Scientific review. - 2015. № 16. - p. 68-71. 